\title{
CLASSICAL FLUID ASPECTS OF NONLINEAR SCHRÖDINGER EQUATIONS AND SOLITONS
}

\author{
James G. Gilson \\ School of Mathematical Sciences \\ Queen Mary College, Mile End Road \\ London E1 4NS, United Kingdom
}

\begin{abstract}
The author cxtends his alternative theory for Schrödinger quantum mechanics by introducing the idea of energy reference strata over configuration space. It is then shown that the vicw from various such strata defines the content of the system of interest and enables a variety of different descriptions of events in the same space time region. Thus according to "the point of view" or energy stratum chosen so the type of Schrödinger equation, linear or otherwise, appropriate to describe the system is determined. A nonlinear information channel between two dimensional fluid action in hyperspace into two dimensional energy hyperspace is shown to exist generally as a background to nonlinear Schrödinger structures. In addition it is shown how soliton solutions of the one dimensional Schrödinger equation are related to two dimensional vortex fields in hyperspace.
\end{abstract}

Key words: Schrödinger, Quantum, Classical Fluids, Solitons, Vorticity.

AMS subject classification: $\quad 81 \mathrm{C} 05,76 \mathrm{C} 05$

\section{INTRODUCTION}

The present author Gilson [1,2,3,4] has developed a theoretical scheme which serves as a "classical" basis for linear Schrödinger quantum theory. In this paper, it will be shown that this same scheme can equally well be used as a generator for the various nonlinear Schrödinger structures and their soliton solutions. This addition to soliton theory $[6,7,8]$ can then be seen as a further connection of the Schrödinger solitons with classical fluid movement. It also emerges that the application of the linear and nonlinear Schrödinger equations, in a sense "simultaneously" to describe the happenings in the same space time region albeit from differing points of view, is a less apparently contradictory pursuit.

The theory developed by the author involves an energy polarized vacuum background which is the source of the influences which cause the system's "quantum" behavior. Thus the question of the "composition" of the background and how it influences 
the system being followed is very important. In the decomposition of the "whole" into identifiable subsystem and its background, the identifiable subsystem should generally be a recognizable physical entity. That usually means that the subsystem should persist as a system "closed", at least with respect to "some" characteristics for some meaningful finite time interval. However, the subsystem may nevertheless be formed from transitory components provided they maintain some "persistent" structural pattern. The "whole" system within a given spatial region means just that; it means the "world" marked off by "given" spatial boundaries at some "time." Of course, not the whole in any absolute sense but rather a region in energy and configuration space for which some form of Schrödinger equation gives a good description. The philosophical complications with such apparently obvious ideas is great and so our definitions of "whole" or "subsystem" can only be regarded as working definitions. It is apparent that the choice of a "physically interesting" subsystem from such a "whole" is likely not to be "unique" and generally it seems that there will be a number of different physically interesting decompositions of the whole into subsystem and background. However, it is also likely that some decompositions may be more important than others. That this is indeed the case will be seen from the examples in the later sections. All these considerations assume a greater importance in systems in which "negative" mass and energy are involved. This is because the decomposition of the "whole" into interesting subsystem and background turns heavily on the question of "where" the background energy ends and the system energy begins in "energy" space and the answer to this question will generally depend on position in configuration space. This issue shows up in a rather less pronounced way in orthodox quantum theory in the appearance of "ground state energy distributions" rather than minimal states of uniformly zero energy over configuration space. A suggestion that arises out of these considerations is that it may be useful to look at decompositions of the "whole" differing from that which is usually employed in orthodox quantum theory. It will be shown in this paper that for systems where the orthodox linear Schrödinger equation is known to give correct physical information, various nonlinear Schrödinger equations can be regarded as representing such alternative decompositions and as one might expect they give alternative and differing information about what is happening in the same "whole" region but in a non contradictory way.

\section{ENERGY STRATA}

Within the alternative theory for "one-dimensional" Schrödinger quantum mechanics, a number of energy carrying functions can be identified. The most important ones are :- 


$$
\left(\mathrm{m}_{0} / 2\right) \mathrm{v}_{1}^{2}, \quad-\left(\mathrm{m}_{0} / 2\right) \mathrm{v}_{2}^{2}, \quad\left(\mathrm{~m}_{0} \mathrm{v} / 2\right) \mathbf{k} \cdot \nabla \wedge \mathbf{v}, \quad \pm \mathrm{m}_{0} \mathrm{c}^{2} \rho^{(0)}
$$

and the related energy density,

$$
E=\mathrm{m}_{\mathrm{O}} \mathrm{c}^{2} \rho^{(0)} \rho^{(3)},
$$

where the velocity field of the monopolar flow is

$$
\mathbf{v}=\mathrm{v}_{1} \mathbf{i}+\mathrm{v}_{2} \mathbf{j}, \quad \mathbf{k}=\mathbf{i}_{\wedge} \mathbf{j}
$$

and $\rho^{(3)}$ is the dipole density per unit three volume.

$$
\rho^{(0)}=v_{0} \rho^{(3)}
$$

is the number of electron rest masses per dipole and $v_{0}=4 \pi 1_{0}^{2} 1_{c}$ is a fixed comparison volume with $\mathrm{cl}_{0}=\mathrm{v}=\mathrm{h} / 2 \mathrm{~m}_{0}$ and $1_{\mathrm{c}}$ the classical electron radius. The formalism is expressed in three dimensions in order to retain physical images of quantities such as the vorticity $\zeta=\nabla_{\wedge} \mathbf{v}$ of the fluid motion over the two dimensional subspace. The quantities quoted above which involve the minus signs always represent "negative" energy monopoles and $\zeta$, the vorticity, can also assume negative values. The detailed explanation of how these various quantities contribute to giving a "classical" explanation for Schrödinger quantum theory can be found in the references Gilson [1,2,3,4]. It is according to how these quantities appear or do not appear in the time evolution energy of the "system" under investigation which determines which type of Schrödinger equation is appropriate for describing the system's movement. One can think of these various energy carrying functions as representing "stratified" energy layers in energy extending through those regions of configuration space which contain the "whole" system. In discussing, this "real" configuration space in the following pages we shall rather freely switch between the real plane image and the complex plane representation with its complex potential for the fluid motion just as can be done in discussing some two-dimensional classical hydrodynamic problems. The currently favoured representation will depend on the aspect we wish to emphasize at any given point in the text. The energy strata are, of course, not generally static but rather variable with space and time and also specific to the actual decomposition of the "whole" being used. The distribution of monopolar energy density $E$ of (2.2) is directly related to an electromagnetic energy density,

$$
E=\mathrm{m}_{0} \mathrm{c}^{2} \rho^{(0)} \rho^{(3)}=(1 / 2)\left(\epsilon_{0} \mathrm{E}_{0}^{2}+\mathrm{B}_{0}^{2} / \mu_{0}\right)
$$




$$
=\mathrm{P}_{0}=\kappa \mathscr{F}_{0} \rho^{(3)},
$$

where $\kappa$ is Boltzman's constant. $E$, as indicated, can be used to define an electromagnetic background pressure $\mathrm{P}_{0}$ and temperature $\mathscr{T _ { 0 }}$. In the alternative theory, the thermal distribution $\kappa \mathscr{T}$ per dipole is always assumed to be present as part of the background. However, on the x-axis, this is just the nonlinear term that appears in the time evolution energy of one of the nonlinear Schrödinger equations. Thus the alternative theory is tailor-made to discuss that nonlinear Schrödinger equation on the $\mathrm{x}$-axis as it stands. The equivalent thermal contribution to that time evolution energy to be used off the $\mathrm{x}$-axis will have to coincide with $\kappa \mathscr{F}$ on the $\mathrm{x}$-axis. The appropriate "feedback" term for the fluid alternative theory is naturally defined by analytic continuation of $\kappa \mathscr{F}$. This last point will be discussed in more detail in the next section.

It is useful to continue the discussion in terms of further thermal interpretation. To this end we introduce a second thermal field $\kappa \mathrm{T}_{1}$ related to the vorticity and defined by,

$$
\begin{aligned}
\kappa \mathrm{T}_{1} & =\mathrm{m}_{0} v \mathbf{k} \cdot \nabla_{\wedge} \mathbf{v}, \\
\text { where } \quad v & =\mathrm{h} / 2 \mathrm{~m}_{0} .
\end{aligned}
$$

If we consider the decomposition that leads to the linear Schrödinger equation, only the "thermal" field $\mathrm{\kappa T}_{1}$ appears in the time evolution energy of the system, while the thermal field $\kappa \mathscr{T}_{0}$ resides in the background. In a decomposition that leads to nonlinear Schrödinger behavior, the real part of the analytically continued $\kappa \mathscr{F}_{0}(x, 0, t)$ appears in the time evolution energy. Thus the distribution of the total thermal energy between the system and background is determined by the actual Schrödinger equation which describes the decomposition. The generation of the thermal fields $\kappa \sigma_{0}$ and $\kappa \mathrm{T}_{1}$ and another thermal field $\kappa \mathscr{F}_{0}(\mathrm{cf})$ from $\kappa \mathscr{F}$ by analytic continuation is classical in the sense that they all satisfy either the Poisson heat equation with a distributed source or they satisfy the Laplace equation. In order to identify the distributed energy source in the Poisson heat equation, we need to consider how these quantum heat fields are generated.

A quantum state at time $t$, say, can arise in two different ways at least Gilson [1]. It could have arrived at the state at time $t$ by a continuous flow process from a state at some earlier time or it could have been created into a running state at time $t$ by an impulsive or discontinuous change over a small time interval $\left(t, t-\tau_{0}\right)$ from the vacuum. Suppose that the momentum field $m_{0}\left(v_{1} \mathbf{i}-v_{2} \mathbf{j}\right)$ which might exist at some time $t$ to have been created by an impulse I over the small time interval $\tau_{0}$ from the vacuum state of zero momentum. Then 


$$
\mathbf{I}=\mathrm{m}_{0}\left(\mathrm{v}_{1} \mathbf{i}-\mathrm{v}_{2} \mathbf{j}\right) \text {. }
$$

The minus sign appears in equation (2.9) because the second component of flow carries negative mass monopoles. The impulse $\mathbf{I}$ creates the velocity field $\mathbf{v}$ and consequently is also responsible for creating the monopolar kinetic energy ,

$$
\begin{gathered}
\mathrm{K}=\left(\mathrm{m}_{0} / 2\right)\left(\mathrm{v}_{1}^{2}-\mathrm{v}_{2}^{2}\right) \\
=\mathrm{K}_{+}+\mathrm{K}_{-} \text {, say }
\end{gathered}
$$

$\mathrm{K}_{+}$being the kinetic energy of the positive mass monopoles and $\mathrm{K}_{-}$that of the negative mass monopoles. The difference of these kinetic energies is

$$
\mathrm{Q}_{0} / 2=\mathrm{K}_{+}-\mathrm{K}_{-}=\left(\mathrm{m}_{0} / 2\right)\left(\mathrm{v}_{1}^{2}+\mathrm{v}_{2}^{2}\right)
$$

and, strangely enough looks more classical than does (2.10), is half the work done by the impulse in producing the kinetic separation or the relative velocity of the opposite monopoles over the xy-plane over the time interval $\tau_{0}$ as though the relative velocity had remained constant over that time. This can be seen by writing (2.12) in the form,

$$
\begin{aligned}
Q_{0} & =\mathbf{I} \cdot\left(\mathbf{v}_{1} \mathbf{i}-\mathrm{v}_{2} \mathbf{j}\right) \\
& =\tau_{0} \overline{\mathbf{v}} \cdot \mathbf{F}_{0}
\end{aligned}
$$

where $\mathbf{F}_{0}$ is the force field equivalent to the impulse $\mathbf{I}$ over the time $\tau_{0}$. Thus

$$
\mathrm{Q}_{0} / \tau_{0}=\overline{\mathbf{v}} \cdot \mathbf{F}_{0}
$$

is the rate of working of the impulse over the time interval $\tau_{0}$. The steps above can be used to make an identification of a local "distributed" rate of heat generation for use in the classical Poisson heat equation as

$$
\mathrm{dQ} / \mathrm{dt}=\mathrm{Q}_{0} / \tau_{0}
$$

in this impulsive situation. The arrival at the form (2.16) by the above steps is only meant to be suggestive and so the reasoning of this section should be regarded as provisional. However, we do arrive at a "correct" equation to describe the various thermal fields. Using (2.16) in the Poisson heat equation, 


$$
\rho \mathrm{dQ} / \mathrm{dt}=\nabla \cdot\left(\sigma^{(\mathrm{th})} \nabla \mathrm{T}\right)
$$

for the case of $\sigma^{(\text {th })}$, the thermal conductivity, constant and in the case of no friction, Laundau and Lifshitz page 185 [11], we get

$$
\begin{gathered}
\rho \mathrm{Q}_{0} / \tau_{0}=\sigma^{(\text {th })} \nabla^{2}(\mathrm{~T}) \\
\text { or } \quad \rho\left(\mathrm{m}_{0} / \tau_{0}\right)\left(\mathrm{v}_{1}^{2}+\mathrm{v}_{2}^{2}\right)=\sigma^{(\text {th })} \nabla^{2}(\mathrm{~T}) .
\end{gathered}
$$

In order that the fundamental temperature distribution $\mathscr{T}_{0}$ be a solution of $(2.18)$ and because $\rho v^{2}=v^{2} \nabla^{2} \rho$ or,

$$
\rho\left(v_{1}^{2}+v_{2}^{2}\right)=c^{2} \nabla^{2}\left(\rho^{(0)} / 1_{3}\right),
$$

where $1_{3}=4 \pi 1_{c}$ and $1_{c}$ is the classical electron radius, from (2.19) and (2.20) it follows that for consistency the thermal conductivity should have the value,

$$
\sigma^{(\mathrm{th})}=\mathrm{k} / \tau_{0} \mathrm{l}_{3}
$$

The electrical conductivity $\sigma$ is given by

$$
\sigma \mu_{0} \mathrm{cl}_{0}=1 .
$$

The relation (2.22) can be arrived at by electromagnetic analysis of the system Gilson [4] or, just for the purposes of the present discussion and because the magnetic diffusivity is equal to $v=c 1_{0}$, we can borrow the same result from magnetohydrodynamics Cowling page 5 (5). Accepting (2.21) and (2.22), it follows that the ratio Sommerfield page 276 [10] of thermal to electrical conductivity in this context should be,

$$
\sigma^{(\mathrm{h})} / \sigma=\kappa^{2} \mathrm{~T}_{0} / \mathrm{e}^{2} .
$$

Apart from a numerical factor, this the Wiedemann-Franz law. We conclude using (2.18) and (2.21) that the thermal fields should be solutions of the classical Poisson equation,

$$
\nabla^{2}(\kappa \mathrm{T})=\mathrm{m}_{0} 1_{3} \rho \mathbf{v}^{2}
$$

The equation (2.24) can be used to formalize the process of separating the "whole" into system of interest and background by specifying various boundary conditions on $T$. Setting boundary conditions on $\mathrm{T}$ is equivalent to selecting a thermal energy stratum over configuration space as a reference level and so generating a unique function $T(x, y, t)$ to be 
the thermal view from that stratum. Clearly, the solutions of (2.24) can have any linear additions which are also solutions to the Laplace equation $\nabla^{2}(\kappa T)=0$. It is these possible linear additions that can be used to fix the solution of (2.24) on some boundary. Before looking at these various points of view defined by the energy strata, the next section will be devoted to considering a particular energy stratum of importance in the case of some nonlinear Schrödinger equations.

\section{FEEDBACK}

"Feedback" is an important aspect of this alternative theory. Even in the case of the "linear" Schrödinger equation it is feedback in the form of the vorticity $\zeta=\nabla_{\wedge} \vee$ that is actually responsible for generating "quantum" behavior. However, this is linear feedback from action space into energy space. The thermal energy,

$$
\kappa \mathscr{T}=m_{0} c^{2} \rho^{(0)}(x, y, t),
$$

is a nonlinear feedback from the fluid complex potential in action space. We shall now examine another energy stratum which results from nonlinear feedback and which seems to be more "fundamental" than (3.1). Let us consider further this question of energy reference levels in relation to local "rotation rates." Local energy can be related to local rotation rates of the dipolar "material" in hyperspace. However, the idea of a uniform condition of local rotation rate zero over all or even just part of configuration space seems to be a geometrical fiction Gilson [1] and should therefore be replaced by some physically defined local rotation rate reference level. This is the same issue of how the "whole" should be decomposed as discussed in the last section. In the case of the linear Schrödinger equation, the author Gilson [1] has shown that the local vacuum fluid vorticity can be used to define a local physical reference level for rotation rates from which the "linear" Schrödinger equation assumes a very simple appearance. Thus the "naturally" occurring vacuum fluid local "angular" velocity provides a special stratum which defines a decomposition with illuminating properties. The existence of such an image simplifying point of view besides indicating closer understanding of the area under examination also prompts the search for other naturally occurring and simplifying viewpoints from which other aspects of Schrödinger type structure might be seen with greater clarity. We shall now establish the existence of just such another "stratum."

The construction of the fluid alternative to Schrödinger quantum theory involves a basic "nonlinear" relation between the fundamental energy distribution function,

$$
\rho^{(0)}(\mathrm{x}, \mathrm{y}, \mathrm{t})=\exp \left(-(\mathrm{i} / 2 \mathrm{v})\left(\omega(\mathrm{z})-\omega^{*}(\overline{\mathrm{z}})\right)\right)
$$


and the complex potential $\omega(\mathrm{z})$ for the "flowing" vacuum.

Here $z^{*}(z)$ denotes the complex conjugate of the functional form only, while $\overline{\omega(z)}$ denotes the full complex conjugate of the function value,

$$
\begin{gathered}
\bar{z}(\mathrm{z})=\mathrm{z}^{*}(\overline{\mathrm{z}}) \\
\text { or } \quad \omega^{*}(\mathrm{x}+\mathrm{iy})=\omega_{1}(\mathrm{x},-\mathrm{y})-\mathrm{i} \omega_{2}(\mathrm{x},-\mathrm{y})
\end{gathered}
$$

The function (3.2) is defined over a real two dimensional Euclidean "hyperspace" and is an information channel between action space in the form of the complex potential $\omega(\mathrm{z})$ for the flowing vacuum and the two dimensional configuration space fundamental energy distribution. (3.2) is in fact just the identity,

$$
\rho^{(0)}(x, y, t) \equiv \exp \left(\ln \rho^{(0)}(x, y, t)\right)
$$

This is because the imaginary part of the complex potential $\omega(\mathrm{z})$, the stream function, turns out to be

$$
\omega_{2}=c 1_{0} \ln \rho^{(0)}(x, y, t) \text {. }
$$

Apart from a numerical factor (3.6) is an entropy per dipole if $\rho^{(0)}(x, y, t)$ is taken to be related to a probability distribution. (3.2) conveys "all" the information in $\omega(\mathrm{z})$ which is a function in action space, via a nonconformal channel, from action space into the definite energy stratum $\mathrm{m}_{0} \mathrm{c}^{2} \rho^{(0)}(\mathrm{x}, \mathrm{y}, \mathrm{t})$ over the real configuration $x y$-plane. Let us consider $\rho^{(0)}(x, 0, t)$, the restriction of $\rho^{(0)}(x, y, t)$ to the $x$ axis. This restriction will usually be differentiable with respect to $\mathrm{x}$ for most values of $\mathrm{x}$. Thus usually it will be possible to analytically continue it back into the complex configuration plane from the $\mathrm{x}$ axis. That is to say, there will be two unique functions,

$$
\begin{aligned}
& \rho_{1}^{(0)}(x, y, t)=\operatorname{Re} \cdot\left(\rho^{(0)}(x+i y, 0, t)\right) \\
\text { and } \quad \rho_{2}^{(0)}(x, y, t) & =\operatorname{Im} \cdot\left(\rho^{(0)}(x+i y, 0, t)\right)
\end{aligned}
$$

resulting from replacing $x$ with $z=x+i y$ in $\rho^{(0)}(x, 0, t)$.

$$
\rho^{(0)}(z, 0, t)=\rho_{1}^{(0)}(x, y, t)+i \rho_{2}^{(0)}(x, y, t)
$$




$$
=\exp \left(-(\mathrm{i} / 2 v)\left(\omega(\mathrm{z})-\omega^{*}(\mathrm{z})\right)\right.
$$

by using (3.2). Consequently

$$
\begin{gathered}
\rho_{1}^{(0)}(x, 0, t)=\rho^{(0)}(x, 0, t), \\
\nabla^{2} \rho_{1}=\nabla^{2} \rho_{2}=0
\end{gathered}
$$

and it is clear that $\mathrm{m}_{0} \mathrm{c}^{2} \rho^{(0)}(\mathrm{z}, 0, \mathrm{t})$ will transform information from action space in the form of the complex potential $\omega(\mathrm{z})$ directly into "complex" energy space in the form of the conjugate pair of harmonic functions $\rho_{1}{ }^{(0)}$. and $\rho_{2}{ }^{(0)}$. In (3.9),

$$
\left(\omega(\mathrm{z})-\omega^{*}(\mathrm{z})\right) / 2 \mathrm{i}
$$

is a "symmetrized" regular function of $\mathrm{z}$ and as such it contains less information than does $\left(\omega(\mathrm{z})-\omega^{*}(\overline{\mathrm{z}})\right) / 2 \mathrm{i}$ which is the imaginary part of $\omega(\mathrm{z})$. The imaginary part of $\omega(\mathrm{z})$ contains as much information as does $\omega(z)$. Thus we infer that the "conformal" transformation (3.9) maps the lesser information contained in (3.12) from action space into complex energy space directly and leaves its mark on that plane in terms of a local polarization energy stratum,

$$
\kappa \mathscr{F}_{0}^{(\mathrm{cf})}=\mathrm{m}_{0} \mathrm{c}^{2} \rho_{1}^{(0)}(\mathrm{x}, \mathrm{y}, \mathrm{t})
$$

Thus (3.13) can be identified mathematically as another energy stratum over the equivalent real xy-plane in which this theory is set. (3.13) is another important energy stratum which can influence the evolution of a system which has been defined by a suitable decomposition. It is thus another candidate for inclusion in the time evolution energy of a system which might be expected to exhibit nonlinear behavior. The main point of this section has been to establish the existence, in real xy-configuration space, of the "lesser" information containing energy stratum (3.13), alongside the dipole rest energy distribution function. The dipole rest energy distribution function (3.5) subsumes the stratum (3.13) as a measure of local vacuum polarization as it contains more information and it is presumably for that reason that it does not appear directly in the theory of the linear Schrödinger equation in hyperspace. However, the stratum $\rho_{1}{ }^{(0)}$ plays a very important role if we change our "point of view" or energy reference level over configuration space as it is on a par with all the other contributions to the time evolution energy in that like the other contributions it is a solution of the Laplace equation (3.11) and it feeds into energy space 
via a conformal channel. The second feedback component (3.8) also plays an important role when the reference energy stratum is changed. It then appears as a contribution to the local polarization process in the source term $\Gamma$ of the continuity equation,

$$
\partial \rho / \partial \mathrm{t}=-\nabla \cdot\left(\rho \mathbf{v}^{(\mathrm{c})}\right)+\Gamma
$$

which describes the flow of density over the real xy-plane. In the next section, it will be shown that the various "views" from different energy strata can be expressed as solutions of the classical Poisson heat equation with specific boundary conditions. The strata $\rho_{1}{ }^{(0)}$ and $\partial v_{2} / \partial x$ play a special role in fixing the boundary conditions.

\section{FIVE CASES}

We have seen that the "possible" thermal energy fields $\kappa T^{(t o t)}$ generated by the "rate" of working of the external impulse I against the relative monopolar displacement over the impulsive state generation time $\tau_{0}$ are solutions of the classical Poisson heat field equation (2.24) or

$$
\nabla^{2}\left(\sigma^{(\mathrm{th})} \mathrm{T}^{(\mathrm{tot})}\right)=\rho \mathbf{v} \cdot \mathbf{F}_{0}
$$

$\kappa T^{(\text {tot })}$ will be taken to be the total "thermal" energy. The actual solution of (4.1) which is used to represent the thermal energy of a given specific system will depend on the boundary conditions of that system. The arbitrariness associated with the solutions of (4.1) arise from the additive modifications of $T^{(t o t)}$ which are solution of (4.1) when the vector $\vee=0$. Such solutions of the resulting Laplace equation can be used as thermal reference levels to set the boundary conditions of $T^{(\text {tot })}$ and consequently define the subsystem or decomposition of the "whole" to which a specific $\mathrm{T}^{(\text {tot })}$ applies as thermal energy. We shall assume that part of the thermal energy which is always present is the "restmass" distribution (3.1) of the dipole field. When this contribution is removed from $\mathrm{kT}^{\text {(tot) }}$ we are left with that part of the thermal energy which will contribute to the time evolution of the "system" while k $\mathscr{F}_{0}$ is relegated to the background. Thus denoting the thermal energy contribution to the time evolution energy $\mathrm{E}^{(\mathrm{tev})}$ by $\mathrm{kT}^{(\mathrm{tev})}$, we have,

$$
\mathrm{T}^{(\text {tev })}=\mathrm{T}^{(\text {tot })}-\mathscr{T} .
$$

Thus the boundary condition on $T^{(t o t)}$ will uniquely define the time evolution thermal energy $\kappa^{(t e v)}$ for the subsystem simultaneously with defining the subsystem. The boundary conditions on $\mathrm{T}^{\text {(tot) }}$ determine an "energy point of view" since they make a definite decomposition of the "whole" into system of interest and the "rest" over some fixed 
space time region. Hence according to the time evolution energy generated by the boundary conditions and the "type" of Schrödinger equation which arises from the time evolution energy so different "aspects" of the "whole" can be analyzed in some region. To illustrate these remarks five possible decompositions of the vacuum into subsystem and background will now be given starting from the boundary conditions on the $y=0$ axis of the two dimensional configuration plane.

The two thermal energies $\kappa \mathscr{F}$ and $\kappa \mathrm{T}_{1}$ given by (3.1) and (2.7) will, on the $\mathrm{y}=0$ boundary, have the forms,

$$
\begin{gathered}
\kappa \mathscr{F}_{0}(\mathrm{x}, 0, \mathrm{t})=\mathrm{m}_{0} \mathrm{c}^{2} \rho^{(0)}(\mathrm{x}, 0, \mathrm{t}) \\
\kappa \mathrm{T}_{1}(\mathrm{x}, 0, \mathrm{t})=-\mathrm{m}_{0} \mathrm{c}^{2} 1_{0}^{2} \partial^{2} \ln \rho^{(0)}(\mathrm{x}, 0, \mathrm{t}) / \partial \mathrm{x}^{2}
\end{gathered}
$$

These functions of $x$ will now be used to fix the boundary value of $T^{(\text {tot })}$, on the $y=0$ axis, for five different decompositions by the following settings :

$$
\begin{array}{ll}
1 & \mathrm{~T}^{(\mathrm{tot})}(\mathrm{x}, 0, \mathrm{t})=2 \mathscr{T}_{0}(\mathrm{x}, 0, \mathrm{t})+\mathrm{T}_{1}(\mathrm{x}, 0, \mathrm{t}) \\
2 & \mathrm{~T}^{(\mathrm{tot})}(\mathrm{x}, 0, \mathrm{t})=\mathscr{T}_{0}(\mathrm{x}, 0, \mathrm{t})+\mathrm{T}_{1}(\mathrm{x}, 0, \mathrm{t}) \\
3 & \mathrm{~T}^{(\mathrm{tot})}(\mathrm{x}, 0, \mathrm{t})=\mathscr{T}_{0}(\mathrm{x}, 0, \mathrm{t}) \\
4 & \mathrm{~T}^{(\mathrm{tot})}(\mathrm{x}, 0, \mathrm{t})=\mathrm{T}_{1}(\mathrm{x}, 0, \mathrm{t}) \\
5 & \mathrm{~T}^{(\text {tot })}(\mathrm{x}, 0, \mathrm{t})=\mathscr{T}_{0}(\mathrm{x}, 0, \mathrm{t})-\mathrm{T}_{1}(\mathrm{x}, 0, \mathrm{t})
\end{array}
$$

The five cases given above by no means exhaust possible decompositions. However, it will be seen that they do represent some significant cases. In general the time evolution energy in hyperspace is given by,

$$
\mathrm{E}^{(\mathrm{tev})}=\operatorname{Re} \cdot(\mathrm{ih} \partial \ln \Psi / \partial \mathrm{t})=\operatorname{Re} \cdot\left(\mathrm{m}_{0} \partial \omega / \partial \mathrm{t}\right)
$$

The forms of the time evolution energy that the five cases lead to in the fluid hyperspace are respectively,

$$
\begin{aligned}
& 1 \mathrm{E}^{(\mathrm{tev})} \mathrm{NLS}^{+}=\left(\mathrm{m}_{0} / 2\right)\left(\mathrm{v}_{1}^{2}-\mathrm{v}_{2}^{2}\right)+\left(\mathrm{m}_{0} \mathrm{v}^{2} / 2\right) \mathbf{k} \cdot \nabla \wedge \mathbf{v}+\mathrm{m}_{0} \mathrm{c}^{2} \mathrm{v}_{0} \operatorname{Re} .(\Psi * \Psi)+\mathrm{V} \\
& 2 \mathrm{E}^{(\mathrm{tev})} \mathrm{S}=\left(\mathrm{m}_{0} / 2\right)\left(\mathrm{v}_{1}^{2}-\mathrm{v}_{2}^{2}\right)+\left(\mathrm{m}_{0} v^{2} / 2\right) \mathbf{k} \cdot \nabla \wedge \mathbf{v}+\mathrm{V} \\
& 3 \mathrm{E}^{(\mathrm{tev})} \mathrm{C}^{-}=\left(\mathrm{m}_{0} / 2\right)\left(\mathrm{v}_{1}^{2}-\mathrm{v}_{2}^{2}\right)+\mathrm{V}
\end{aligned}
$$




$$
\begin{array}{ll}
4 & \mathrm{E}^{(\mathrm{tev})} \mathrm{NLS}^{-}=\left(\mathrm{m}_{0} / 2\right)\left(\mathrm{v}_{1}^{2}-\mathrm{v}_{2}^{2}\right)+\left(\mathrm{m}_{0} v^{2} / 2\right) \mathbf{k} \cdot \nabla \wedge \mathbf{v}-\mathrm{m}_{0} \mathrm{c}^{2} \mathrm{v}_{0} \operatorname{Re} \cdot(\Psi * \Psi)+\mathrm{V} \\
5 & \mathrm{E}^{(\mathrm{tev})} \mathrm{NLS}^{\prime}=\left(\mathrm{m}_{0} / 2\right)\left(\mathrm{v}_{1}^{2}-\mathrm{v}_{2}^{2}\right)-\left(\mathrm{m}_{0} v^{2} / 2\right) \mathbf{k} \cdot \nabla \wedge \mathbf{v}+\mathrm{V}
\end{array}
$$

The time evolution thermal field, $\mathrm{T}_{\mathrm{NLS}}+$, for the nonlinear Schrödinger equation with the plus sign is related to the conformal feedback field by

$$
\mathrm{T}^{(\mathrm{tev})} \mathrm{NLS}^{+}=\mathrm{T}_{0}{ }^{(\mathrm{cf})}+\mathrm{T}_{1}
$$

Thus if we use the thermal field for the nonlinear Schrödinger equation with the plus sign for comparison of the various cases, $\mathrm{T}^{(\text {tev })}$ can be expressed as $\mathrm{T}_{\mathrm{NLS}}+$ relative to $\mathrm{T}^{\text {(ref) }}$ as in (4.9), where generally $\mathrm{T}^{(\mathrm{tev})}=\mathrm{T}_{\mathrm{NLS}}{ }^{+}-\mathrm{T}^{(\mathrm{rcf})}$ and in the various cases :

$$
\begin{aligned}
& 1 \mathrm{~T}^{(\mathrm{tev})}=\mathrm{T}_{\mathrm{NLS}^{+}}-0 \\
& 2 \mathrm{~T}^{(\mathrm{tev})}=\mathrm{T}_{\mathrm{NLS}^{+}}-\mathscr{T}_{0}{ }^{(\mathrm{cf})} \\
& 3 \mathrm{~T}^{\text {(tev) }}=\mathrm{T}_{\mathrm{NLS}^{+}}-\left(\mathscr{F}_{0}^{(\mathrm{cf})}+\mathrm{T}_{1}\right) \\
& 4 \mathrm{~T}^{(\mathrm{tev})}=\mathrm{T}_{\mathrm{NLS}^{+}}-2 \mathscr{F}^{(\mathrm{cf})} \\
& 5 \mathrm{~T}^{(\text {tev })}=\mathrm{T}_{\mathrm{NLS}^{+}}-\left(\mathscr{F}_{0}^{(\mathrm{c})}+2 \mathrm{~T}_{1}\right)
\end{aligned}
$$

with the last subtracted term being the reference level for that particular case. However one should not forget that the various named functions are entirely different functions in the five different cases.

The Schrödinger equations which are generated by these various time evolution energies, on the $y=0$ axis, are in the five cases respectively:

$$
\begin{aligned}
& 1 \text { ih } \partial \Psi / \partial \mathrm{t}=-\left(\AA^{2} / 2 \mathrm{~m}_{0}\right) \partial^{2} \Psi / \partial \mathrm{x}^{2}+\mathrm{m}_{0} \mathrm{c}^{2} \mathrm{v}_{0} \Psi * \Psi^{2}+\mathrm{V} \Psi \\
& 2 \mathrm{ih} \partial \Psi / \partial \mathrm{t}=-\left(\hbar^{2} / 2 \mathrm{~m}_{0}\right) \partial^{2} \Psi / \partial \mathrm{x}^{2}+\mathrm{V} \Psi \\
& 3 \mathrm{i} h \partial \Psi / \partial \mathrm{t}=-\left(\AA^{2} / 2 \mathrm{~m}_{0}\right) \partial^{2} \Psi / \partial \mathrm{x}^{2}+\mathrm{m}_{0} \mathrm{v}^{2}\left(\partial^{2} \ln (\Psi * \Psi) / \partial \mathrm{x}^{2}\right) \Psi+\mathrm{V} \Psi \\
& 4 \mathrm{ih} \partial \Psi / \partial \mathrm{t}=-\left(h^{2} / 2 \mathrm{~m}_{0}\right) \partial^{2} \Psi / \partial \mathrm{x}^{2}-\mathrm{m}_{0} \mathrm{c}^{2} \mathrm{v}_{0} \Psi * \Psi^{2}+\mathrm{V} \Psi \\
& 5 \text { ih } \partial \Psi / \partial \mathrm{t}=-\left(h^{2} / 2 \mathrm{~m}_{0}\right) \partial^{2} \Psi / \partial \mathrm{x}^{2}+\mathrm{m}_{0} \mathrm{v}^{2} 2\left(\partial^{2} 1 \mathrm{n}(\Psi * \Psi) / \partial \mathrm{x}^{2}\right) \Psi+\mathrm{V} \Psi
\end{aligned}
$$

In the equations (4.10), $\mathrm{v}_{0} \Psi * \Psi$ is dimensionless with $\mathrm{v}_{0}$ the standard comparison "volume." The first of these equations arises from $\mathrm{E}_{\mathrm{NLS}^{+}}$and is the nonlinear Schrödinger equation with the positive sign before the cubic term. The second equation 
arises from $\mathrm{E}_{\mathrm{S}}$ and is the usual linear Schrödinger equation. The third arises from $\mathrm{E}_{\mathrm{C}^{-}}$ and is a nonlinear Schrödinger equation which can be associated with a classical dynamical motion because in this case there is effectively no feedback from action space but this particular equation still involves a second component velocity field carrying negative mass. The fourth which arises from $\mathrm{E}_{\mathrm{NLS}^{-}}$is the nonlinear Schrödinger equation with the negative sign before the cubic term. Finally, the fifth equation which arises from $\mathrm{T}_{N L S^{\prime}}$ is another type of nonlinear Schrödinger equation which in the special case of the external potential $\mathrm{V}$ being zero has at least one solution in common with the equation of case four. To complete this section, we shall now look at a rather simple example to show how the "hyperspace view" of one of the nonlinear Schrödinger equations can help to give an extended perspective on fluid aspects of soliton theory $[6,7,8]$.

Consider the well known one soliton solution of the nonlinear Schrödinger equation of case 4. The wave function for this case has the form,

$$
\Psi=\lambda \operatorname{sech}\left(\mathrm{A}_{0}+\alpha_{1} \mathrm{x}+\alpha_{2} \mathrm{t}\right) \exp \left[\mathrm{i}\left(\mathrm{B}_{0}+\beta_{1} \mathrm{x}+\beta_{2} \mathrm{t}\right)\right] .
$$

It will be sufficient to take the special but non trivial case

$$
\begin{aligned}
& \mathrm{A}_{0}=\alpha_{2}=\mathrm{B}_{0}=\beta_{1}=0 \\
& \text { with } \alpha_{1}=1 / 2 \mathrm{l}_{0} \text { and } \beta_{2}=\mathrm{c} / 4 \mathrm{l}_{0}
\end{aligned}
$$

Then the wave function can be taken to be

$$
\Psi=\lambda \operatorname{sech}\left(\mathrm{x} / 2 \mathrm{l}_{0}\right) \exp \left(\mathrm{ict} / 41_{0}\right)
$$

The associated fluid complex potential in hyperspace,

$$
\begin{aligned}
& \text { when } \quad \mathrm{x} \rightarrow \mathrm{z}=\mathrm{x}+\mathrm{iy} \quad \text { is } \\
& \omega(\mathrm{z}, \mathrm{t})=2 \mathrm{icl}_{0} \ln \Psi(\mathrm{z}, \mathrm{t}) \\
& =2 \mathrm{icl}_{0}\left(\ln \left(\operatorname{sech}\left(\mathrm{z} / 2 \mathrm{1}_{0}\right)\right)+\mathrm{ict} / 4 \mathrm{I}_{0}+\ln \lambda\right)
\end{aligned}
$$

However, we find, by using an argument similar to that given in Milne-Thomson page 377 [9] for another case, that the complex potential for an infinite row of vortices each of strength $r$ in the complex plane separated by the distance a and at positions given by,

$$
\pm a i / 2, \quad \pm 3 a i / 2, \quad \ldots \ldots \ldots . . \quad \pm(2 n+1) \text { ai } / 2 \quad \ldots \ldots .
$$

has the form, 


$$
\omega(\mathrm{z}, \mathrm{t})=-i \ln (\operatorname{sech}(\pi \mathrm{z} / \mathrm{a}))+\mathrm{g}(\mathrm{t})
$$

where $g(t)$ is some function of time. If we take

$$
\mathrm{r}=-2 \mathrm{cl}_{0} \quad \text { and } \quad \mathrm{a}=2 \pi \mathrm{l}_{0}
$$

(4.15) and (4.16) agree. The time dependent term $-c^{2} t / 2$ gives the eigenvalue of the energy associated with the solution (4.14) when used in the formula (4.6) for the time evolution energy,

$$
\mathrm{E}^{(\mathrm{tev})}=\operatorname{Re} \cdot\left(\mathrm{m}_{0} \partial \omega(\mathrm{z}) / \partial \mathrm{t}\right)=-\mathrm{m}_{0} \mathrm{c}^{2} / 2
$$

which is the "negative" energy eigenvalue of the one soliton solution being used. Thus the single soliton on the $\mathrm{x}$-axis is one cross section of the hyperspace solution in which an infinite row of two dimensional vortices "exist" in the vacuum fluid. The "soliton" on the $\mathrm{x}$-axis lies midway between the vortex above it and below it in the complex plane and at all such mid positions of consecutive vortices in the infinite row there are more cross sections parallel with the $\mathrm{x}$-axis which can also be interpreted as one dimensional "solitons." It seems that it is the more extensive and interesting fluid system consisting of two dimensional vortices that generates the primary flow pattern in hyperspace and this only incidentally leads to the somewhat "degenerate" object on the real axis which is designated "a soliton."

\section{CONCLUSIONS}

The fluid vacuum polarization hyperspace theory which was originally proposed as a classical alternative to linear Schrödinger quantum theory can also be seen to encompass the nonlinear Schrödinger structures "simultaneously." The alternative theory is fundamentally nonlinear but is comformably transformed into the linear quantum theory wave function $\Psi$ by the nonlinear transformation (5.1).

$$
\Psi(\mathrm{z}, \mathrm{t})=\exp (-\mathrm{i} \omega(\mathrm{z}, \mathrm{t}) / 2 \mathrm{v})
$$

from the fluid action space complex potential. However, by changing energy reference levels or strata over configuration space the consequent changed time evolution energy leads to a changed wave function and a changed complex potential which are such that the same conformal nonlinear transformation (5.1) above, instead of linearizing the theory, reduces the theory in some cases to quadratic nonlinear in feedback energy or cubic nonlinear feedback in the Schrödinger equation and in other cases reduces the theory to more complicated nonlinear differentiated logarithmic terms in the Schrödinger equation. 
Important advantages of this alternative fluid model of quantum theory are its generality and its capacity to invoke a vast range of classical images which can be used to "explain" many rather abstract features that appear in the orthodox version of Schrödinger quantum mechanics. The work in this paper has shown that this same theory can also be used "simultaneously" to exploit the insight that can be obtained from a large range of the nonlinear structures that seem to be gaining increasing importance in the study of natural phenomena.

Acknowledgement: I am very grateful to Professor Sir Michael Atiyah for some motivating ideas and in particular for drawing my attention to the general need to incorporate nonlinear Schrödinger theory into any attempt to reformulate quantum mechanics. 


\section{REFERENCES}

[ 1] Gilson, J.G., The nature of a quantum state, Acta Physica Hungarica, 60 (3-4), pp 145-160 (1986).

[2] Gilson, J.G., An alternative view of the Schrödinger Process, Speculations in Science and Technology, Vol 9, (1986)

[3] Gilson, J.G., Six-dimensional flow and the three dimensional Schrödinger equation, Journal of Mathematical and Physical Sciences, Vol 20, No 1, pp 55-63. Feb. (1986)

[4] Gilson, J.G., A real plane representation of the electrodynamics of the complex Schrödinger configuration plane, Annals de la Fondation Louis de Broglie, Vol 6, No 1, (1981)

[5] Cowing, T.G., Magnetohydrodynamics, Adam Hilger (1976)

[ 6] Bullough, R.K., Caudry P.G., Topics in Current Physics, Solitons, SpringerVerlag Berlin, (1980)

[7] Rebbi, C., Soliani, G., Solitons and Particles, World Scientific (1984)

[ 8] Drazin, P.G., Solitons, Lecture note series 85, London Mathematical Society, Cambridge University Press (1983)

[ 9] Milne-Thomson, Theoretical Hydrodynamics, Macmillan and Co Ltd, New York (1949)

[10] Sommerfield, A., Thermodynamics and Statistical Mechanics, Vol 5, Academic Press (1967)

[11] Landau and Lifshitz, Course of Theoretical Physics, Vol 6, Pergamon Press (1981) 


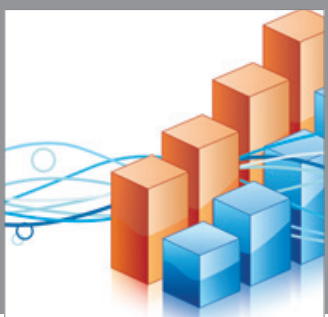

Advances in

Operations Research

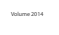

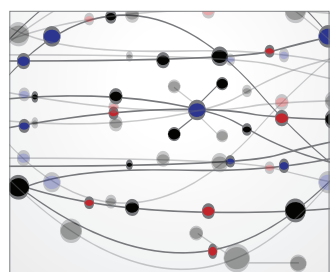

\section{The Scientific} World Journal
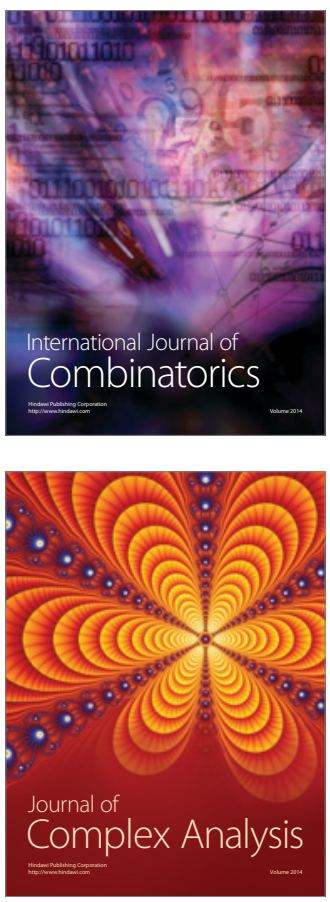

International Journal of

Mathematics and

Mathematical

Sciences
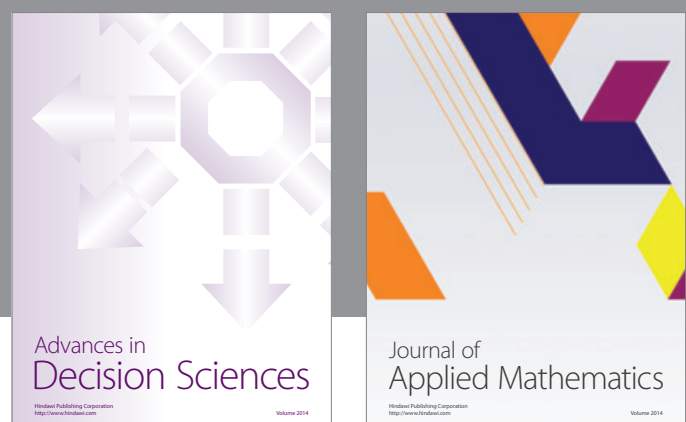

Journal of

Applied Mathematics
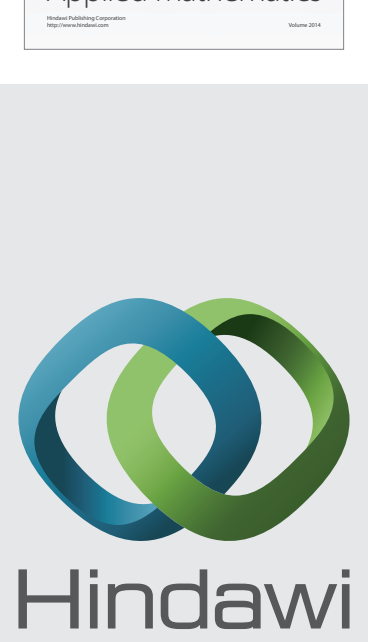

Submit your manuscripts at http://www.hindawi.com
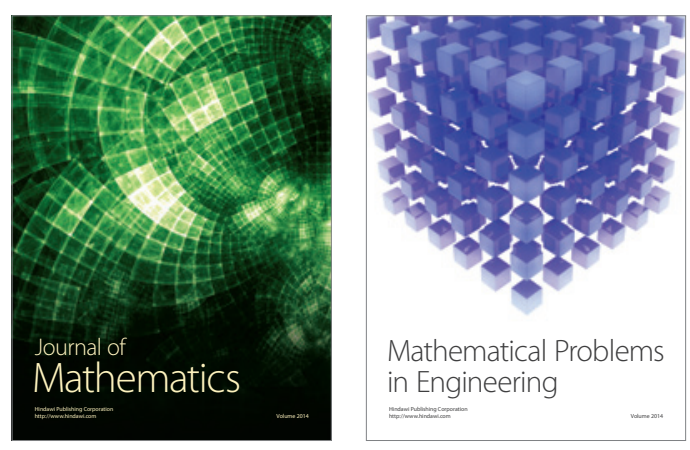

Mathematical Problems in Engineering
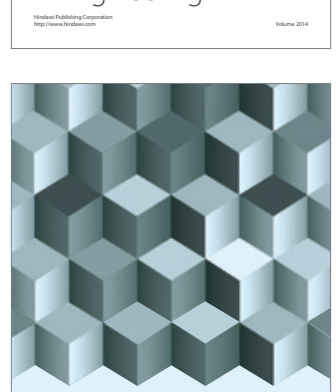

Journal of

Function Spaces
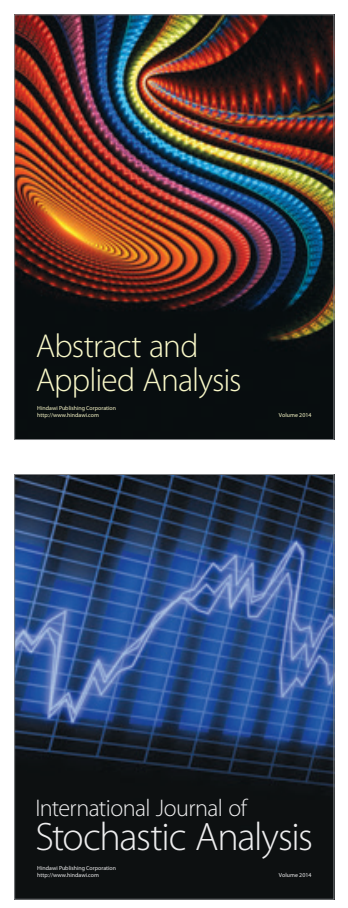

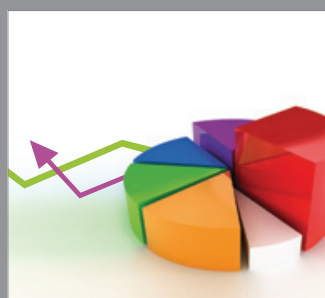

ournal of

Probability and Statistics

Promensencen
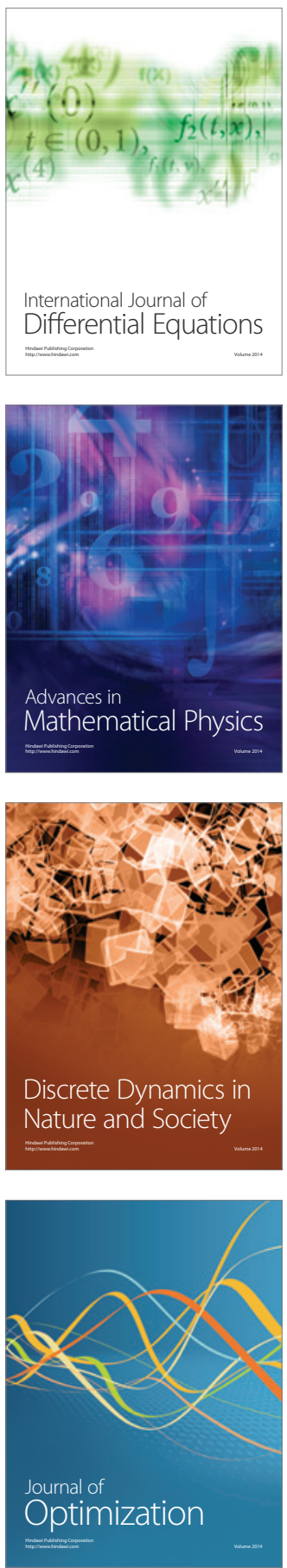